\title{
Comportamentos de amamentação e mamada em bubalinos, suas relações com período de lactação, idade e sexo dos bezerros
}

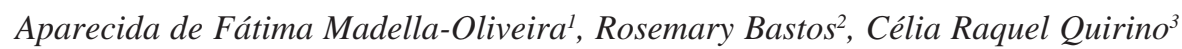

\section{RESUMO}

Objetivou-se neste trabalho investigar a frequência e a duração dos tipos de amamentações durante o período de lactação em fêmeas bubalinas e os tipos de mamadas em relação à idade e ao sexo dos bezerros. Trinta e cinco fêmeas e seus bezerros foram observados durante três dias consecutivos a cada mês, ao longo de nove meses. A amamentação foi caracterizada como: amamentação filial isolada (AFI), amamentação filial coletiva (AFC) e amamentação não-filial (ANF). As mamadas executadas pelos bezerros foram caracterizadas como: mamada filial isolada (MFI), mamada filial coletiva na própria mãe (MFC1), mamada filial coletiva em outra fêmea (MCF2) e mamada não-filial (MNF). Os dados foram analisados pela análise de variância, e as médias foram comparadas pelo teste Tukey, considerando $\mathrm{P}<0,05$. A AFI apresentou frequência e duração maiores do que os outros tipos de amamentações $(\mathrm{P}<0,05)$, sendo predominante em quase todo o período de lactação. A frequência da MFI foi alta, principalmente no primeiro mês. A duração foi maior quando comparada aos outros tipos de mamadas até o quinto mês $(\mathrm{P}<0,05)$. Em relação ao sexo dos bezerros não foram encontradas diferenças significativas $(\mathrm{P}>0,05)$ para as médias da frequência e a duração dos tipos de mamadas. Os resultados mostram que as fêmeas bubalinas têm maior aptidão inata para a AFI em relação aos outros tipos de amamentações, predominando durante quase todo o período de lactação. Os bezerros bubalinos são mais dependentes da MFI durante os primeiros cinco meses de idade, e o sexo dos bezerros não influenciou nos tipos de mamadas.

Palavras-chave: Aloamamentação, duração, frequência, bubalus bubalis.

\section{ABSTRACT}

\section{Nursing and sucking behaviour in buffalos in relation to lactation period, age and calf sex}

The objectives of the present study were to investigate the frequency and duration of nursing behaviour in female buffalos during the lactation period and the types of suckling behaviour in relation to age and calf sex. Thirty-five females with their calves were observed during three consecutive days in each month, over a period of nine months. The nursing behaviour was characterized as isolated filial nursing (IFN); collective filial nursing (CFN) and nonfilial nursing (NFN). The suckling behaviour was classified as isolated filial suckling (IFS); collective filial suckling on their own mothers (CFS1); collective filial suckling on other females (CFS2) and nonfilial suckling (NFS). Data were analyzed by analysis of variance and means were compared by the Tukey test considering $\mathrm{P}<0,05$. IFN showed frequency and duration higher than the other types of the nursing $(\mathrm{P}<0,05)$, with predominance in almost all period of lactation. The frequency of IFS was high mainly in the first month and the duration of IFS was higher than other types of suckling until the fifth month $(\mathrm{P}<0,05)$ In relation to calf sex, no significant differences $(\mathrm{P}>0,05)$ were found for the means of frequency and duration of the types of sucking. The results show that the buffalo females have larger innate aptitude

\footnotetext{
Recebido para publicação em novembro de 2007 e aprovado em janeiro de 2010

${ }^{1}$ Bióloga, Doutora. Instituto Federal de Educação, Ciência e Tecnologia do Espírito Santo, Campus de Alegre,Rodovia Cachoeiro, Km 48, Caixa Postal 47, Distrito de Rive, 29500000, Alegre, Espírito Santo, Brasil. madellabio@gmail.com

${ }^{2}$ Farmacêutica-Bioquímica, Doutora. Universidade Estadual do Norte Fluminense Darcy Ribeiro, Laboratório de Melhoramento Genético Animal, Av Alberto Lamego, 2000, CCTA/LMGA/Setor de Fisiologia Animal, Pq Califórnia, 28013-642, Campos dos Goytacazes, Rio de Janeiro, Brasil. rosebast@gmail.com

3 Engenheira agrânoma, Doutora. Universidade Estadual do Norte Fluminense Darcy Ribeiro, Laboratório de Melhoramento Genético Animal, Av Alberto Lamego, 2000, CCTA/ LMGA/Setor de Fisiologia Animal, Pq Califórnia, 28013-642, Campos dos Goytacazes, Rio de Janeiro, Brasil. crq@uenf.br
} 
for IFN in relation to the other types of nursing during almost all the lactation period. The calves are more dependent on IFS during the first five months and the calf sex did not influence the types of suckling behaviour.

Key words: Allonursing, duration, frequency, Bubalus bubalis.

\section{INTRODUÇÃO}

O comportamento parental é definido como qualquer comportamento que inclui todas as atividades dos pais dirigidas à descendência, com a finalidade de garantir sua sobrevivência e seu crescimento (Brown, 1998). Em mamíferos, o cuidado infantil primário é da mãe, chamado comportamento materno. Entretanto, em alguns mamíferos, o pai provê quantidades significativas do cuidado infantil, o que pode ser chamado comportamento paterno (Smith, 1977).

Nos mamíferos, o início da formação dos laços materno-filiais tem sido tradicionalmente considerado como ocorrendo após o parto (Paranhos da Costa \& Andriolo, 1998). No búfalo, acredita-se que o estabelecimento do vínculo entre a mãe e o bezerro se dê logo nas primeiras horas de vida. O processo de reconhecimento da cria pela mãe sofre forte influência de uma série de comportamentos da mãe e do bezerro, destacando-se a ingestão de fluidos amnióticos e membranas fetais que, provavelmente, auxiliam nesse processo. O odor e o paladar desses fluidos e membranas são importantes, porque a mãe aprende a conhecer o próprio bezerro (Paranhos da Costa \& Andriolo, 1998).

O principal componente dos cuidados parentais entre os mamíferos é a amamentação, pois os neonatos dependem de um suprimento adequado de leite secretado das glândulas mamárias da mãe por um período de tempo após o parto (Blass \& Teicher, 1980). Além disso, uma fêmea adulta pode permitir que filhotes que não o seu mamem, sendo este comportamento um exemplo de comportamento aloparental, denominado como aloamamentação, não sendo relacionado com o grau de parentesco ou relações recíprocas (Riedman, 1982, Hoogland et al., 1989).

O comportamento aloparental no aspecto nutricional foi identificado em 120 espécies de mamíferos, frequentemente em primatas, e em 150 espécies de pássaros que pertencem às mais diversas ordens (Riedman, 1982). Esse comportamento foi observado em várias espécies de mamíferos, entre elas elefante-marinho (Mirounga angustirostris) (Riedman \& Le Boeuf, 1982); cão-depradaria (Cynomys ludovicianus) (Hoogland et al., 1989); capivara (Hydrochoerus hydrochaeris) (Nogueira et al.,
2000); veado (Cervus elaphus hispanicus) (LandeteCastillejos et al., 2000), bovinos (Bos taurus) (Víchová \& Bartoš, 2005) e búfalos (Bubalus bubalis) (Tulloch, 1979; Murphey et al., 1991 e 1995; Andriolo, 1995; Paranhos da Costa \& Andriolo, 1998; Paranhos da Costa et al., 2000; Andriolo et al., 2001; Bastos et al., 2002).

Em búfalos o comportamento de aloamamentação foi relatado pela primeira vez por Tulloch (1979) em animais da raça Carabao, observando que, após a morte de algumas fêmeas bubalinas, os órfãos tentaram mamar em outras fêmeas, obtendo sucesso. Murphey et al. (1991) verificaram que as fêmeas bubalinas solicitadas com mais frequência por seus próprios bezerros e por bezerros alheios demonstraram maior tendência em aceitá-los. Os bezerros bubalinos que realizaram a aloamamentação, segundo Murphey et al. (1995), tiveram esse comportamento devido ao fornecimento insuficiente de leite das mães. Por consequência, eles diminuíram as tentativas de amamentação em sua própria mãe, possivelmente por ela permitir a amamentação não-filial, devido à falta de experiência materna.

Os objetivos deste estudo foram verificar a frequência e a duração dos tipos de amamentação durante o período de lactação em fêmeas bubalinas, e os tipos de mamada em relação à idade e ao sexo dos bezerros.

\section{MATERIAL E MÉTODOS}

O trabalho experimental foi realizado na Fazenda Cataia, situada no município de São Francisco do Itabapoana, Estado do Rio de Janeiro, localizada na latitude sul $21^{\circ} 18^{\prime}$ $07^{\prime \prime}$ e longitude oeste $40^{\circ} 57^{\prime} 41^{\prime \prime}$, a uma altitude de $8 \mathrm{~m}$, aproximadamente, apresentando topografia com leves ondulações, com presença de várzeas nas partes mais baixas, sendo o solo predominantemente arenoso.

No sistema de produção existe criação de búfalos (Bubalus bubalis) mestiços das raças Murrah e Mediterrâneo, com o objetivo de abate dos machos aos dois anos de idade e produção de leite.

Foram utilizadas 35 fêmeas e seus respectivos bezerros, provenientes de um rebanho de, aproximadamente, 200 animais. A idade das fêmeas variarou de três a 12 anos. O grupo foi composto por duas fêmeas primíparas e 33 
multíparas. Três touros permaneceram durante todo o ano com as fêmeas, mas o maior número de monta ocorreu nos meses de maio a julho. Os bezerros usados nos experimentos nasceram nos meses de março (14), abril (nove) e maio (12).

Foram colocados brincos de plásticos, nos animais para que eles ficassem com identificação permanente; as fêmeas receberam número diferente do dos bezerros. A marcação dos bezerros e das fêmeas foi realizada com números ordinários na anca, utilizando tinta óleo de cor amarela, uma vez a cada mês, antes das observações.

No manejo da fazenda, após o parto a mãe e o bezerro permaneceram durante 15 dias no pasto da maternidade, separados das demais fêmeas e, após esse período, foram levados para o curral, quando se iniciava a ordenha. Essa era manual, com o bezerro ao pé, e iniciava às $04 \mathrm{~h}$. Ao final da ordenha, em torno de $06 \mathrm{~h}$, o rebanho era levado para o pasto, onde as fêmeas permaneciam juntas com seus bezerros até as $12 \mathrm{~h}$. O rebanho era recolhido às $12 \mathrm{~h}$, quando havia apartação de adultos e jovens em diferentes pastos.

As observações ocorreram a cada mês durante três dias consecutivos, de maio de 2002 a janeiro de 2003. A forma de observação foi direta e contínua (Martin \& Bateson, 1986), sendo iniciada às 06 h e encerrada às 12 h, totalizando 162 horas.

Os animais foram observados, a cavalo, durante todo o período em que estavam soltos no pasto. Utilizou-se um binóculo para identificação dos números a certa distância, com o objetivo de não interferir no comportamento. Foi usado relógio digital para os registros dos horários das mamadas, seguindo-se a identificação da mãe e do bezerro envolvidos e foram marcados o início e o término do comportamento da mamada, que foram registrados em um gravador de áudio.

As mamadas foram caracterizadas a partir do momento em que o bezerro fixa sua boca no teto da fêmea, fazendo movimentos de sucção. As amamentações e as mamadas foram classificadas em categorias para as fêmeas e bezerros, segundo Andriolo (1995). Para as fêmeas observaram-se: 1- amamentação filial isolada (AFI), quando a fêmea amamentava o seu próprio bezerro; 2- amamentação filial coletiva (AFC), quando uma fêmea iniciava a amamentação de seu próprio bezerro e durante esse episódio um e, ou, mais bezerros iniciavam a mamada ao mesmo tempo; 3-amamentação não-filial isolada (ANFI), quando uma fêmea amamentava apenas um bezerro que não é o seu; 4- amamentação não-filial coletiva (ANFC), quando uma fêmea amamentava mais de um bezerro ao mesmo tempo e nenhum deles é o seu e; 5- amamentação nãofilial (ANF = ANFI + ANCF), soma das amamentações não-filiais. Para os bezerros: 1- mamada filial isolada (MFI), quando o bezerro mamou sozinho em sua mãe; 2- mamada filial coletiva, que foi dividida em: a - (MFC1) quando o bezerro mamava em sua mãe acompanhado de outros bezerros e b - (MFC2) quando o bezerro mamou em outra fêmea e o bezerro dela participou do evento em algum momento e; 3- mamada não-filial (MNF), quando os bezerros mamaram em uma fêmea cujo bezerro não tenha participado em nenhum momento.

Para as amamentações cedidas pelas fêmeas e as mamadas executadas pelos bezerros foram calculados: a frequência e o tempo de duração com que as fêmeas e os bezerros realizaram tais comportamentos. A frequência foi expressa em episódios/dia/pasto e a duração em segundos/dia/pasto.

Para a análise estatística, os dados de comportamento (frequência e duração das diferentes categorias de mamadas) foram transformados por $(\mathrm{X}+1 / 2)^{1 / 2}$, de acordo com Martin \& Bateson (1986). Na análise de variância, para frequência e duração para as fêmeas e bezerros consideraram-se os efeitos fixos do mês de observação, tipo de mamada, a idade e o sexo do bezerro (PROC. GLM, SAS, 1996). As médias foram comparadas pelo teste Tukey, e consideraram-se os valores $\mathrm{P}<0,05$.

\section{RESULTADOS E DISCUSSÃO}

Verificou-se diferença significativa $(\mathrm{P}<0,0001)$ para os diferentes tipos de amamentação cedida pelas fêmeas bubalinas (frequência e duração). Destaca-se a magnitude do tipo da amamentação filial para a frequência (0,86 \pm 0,35 episódio/dia/pasto) em relação aos outros tipos de amamentação durante os nove meses de observação. Ocorreram em menor proporção a amamentação coletiva (0,69 \pm 0,23 episódio/dia/pasto) e a não-filial ( $0,66 \pm 0,19$ episódio/dia/pasto), que não apresentaram diferenças entre si $(\mathrm{p}>0,05)$.

As fêmeas bubalinas gastaram mais tempo na amamentação filial isolada (8,34 $\pm 10,23$ segundos/dia/ pasto) do que na coletiva filial (2,32 $\pm 5,16$ segundos/dia/ pasto) e não-filial (0,93 $\pm 1,55$ segundo/dia/pasto). A frequência e a duração apresentaram valores baixos quando comparados com Bastos, (2000) e Andriolo et al. (2001). Isso pode ser devido à forma do manejo adotada na fazenda, em que durante todo o período da ordenha os bezerros permaneceram juntos com as suas mães no curral, realizando a amamentação, acarretando diminuição das amamentações no pasto. Os resultados estão de acordo com a literatura (Tulloch, 1979; Murphey et al., 1991 e 1995; Andriolo, 1995; Bastos, 2000; Bastos et al., 2002), nos quais verificou-se a incidência do comportamento de amamentação coletiva para a espécie bubalina em diferentes tipos de manejo.

Os valores da AFI, tanto para a frequência com que as fêmeas bubalinas amamentaram quanto para o tempo em 
que se dispuseram a essa amamentação, foram maiores do que os da AFC e ANF. Esses resultados estão de acordo com Bastos (2000), contrastando com Andriolo et al. (2001), os quais observaram que a ocorrência e a duração da amamentação filial isolada foram inferiores à da amamentação coletiva filial. E a amamentação não-filial mostrouse bem reduzida durante os meses de observação. Os baixos valores para a amamentação coletiva podem sugerir que o manejo da fazenda interferiu neste comportamento e, segundo Paranhos da Costa e Andriolo (1998), a amamentação coletiva varia em função das condições ecológicas e/ou da criação.

A frequência e duração em relação aos tipos de amamentação nos meses de lactação foram significativas $(\mathrm{P}<0,05)$. A frequência da AFI foi predominante em relação aos outros tipos de amamentações até o sétimo mês de lactação e, a partir desse período, as mães desses bezerros iniciaram o desmame natural. As frequências da AFC e da ANF foram constantes durante todo o período de lactação, não apresentando diferenças entre si (p > 0,05) (Tabela 1).

Para a duração das amamentações, verificou-se declínio acentuado conforme aumentou o período de lactação. A duração da AFI foi predominante em relação à AFC e ANF em quase todo o período de lactação das fêmeas bubalinas. A duração da AFC se apresentou constante ao longo do período de lactação e os valores da ANF apresentaram-se baixos na fase de aleitamento (Tabela 2). Esses resultados estão de acordo com Bastos (2000) e Andriolo et al. (2001), que evidenciam a importância da amamentação filial para a espécie bubalina. Segundo Andriolo et al. (2001), a AFI é substituída pela ACF, que ainda privilegia a amamentação do próprio bezerro. Essa substituição do tipo de amamentação iniciou-se a partir do segundo mês de lactação, evidenciando o investimento materno no seu pró- prio bezerro, sendo a amamentação coletiva; no caso, um oportunismo do bezerro que tenta maximizar seu alimento.

A frequência e a duração dos diferentes tipos de mamadas executadas pelos bezerros em relação à idade diferiram $(\mathrm{P}<0,05)$. A frequência das mamadas executadas pelos bezerros foi alta no primeiro mês para a MFI (Tabela 3), comprovando sua importância e, após esse período, ocorre decréscimo, que foi acentuado no sétimo mês, estabelecendo a desmama natural. A frequência com que os bezerros procuraram realizar a MFC1, MFC2 e MNF foi constante durante todos os meses (Tabela 3), não apresentando diferenças significativas entre si $(P>0,05)$. Esses resultados estão de acordo com Andriolo (1995), que, em seus estudos, relata a suma importância da mamada filial no primeiro mês.

A Tabela 4 mostra que a duração da MFI apresentou valores superiores até o quinto mês em relação aos outros tipos de mamada, tendo no sexto mês a duração da MFI apresentado diferença significativa com MCF1 e MNF $(\mathrm{P}<0,05)$ e no sétimo mês a duração da MFI não teve diferença entre as médias com a MFC1 ( $\mathrm{P}>0,05)$. A duração da MFC1 e da MNF mantive-se constante durante toda a idade dos bezerros (Tabela 4). A duração da MNF apresentou baixos valores, sugerindo a pouca importância dessa mamada para os bezerros.

Esses resultados evidenciaram a motivação dos bezerros em mamar em sua própria mãe, isso devido ao reconhecimento e pelos cuidados maternais da fêmea com o bezerro, o que está de acordo com Andriolo et al. (2001), os quais observaram que a motivação exclusiva pela própria mãe não variou ao longo do período, sugerindo que, além de haver um reconhecimento da fêmea pelo bezerro, o seu interesse em mamar nela permaneceu constante. Além disso, a partir do crescimento dos bezerros e com a diminuição da produção de leite de suas

Tabela 1. Médias e respectivos desvios-padrão da freqüência (episódios/dia/pasto) das amamentações cedidas pelas fêmeas para os diferentes tipos de amamentação, em relação aos meses de lactação. (AFI - amamentação filial isolada; AFC - amamentação filial coletiva e ANF - amamentação não-filial)

\begin{tabular}{lccc}
\hline & \multicolumn{3}{c}{ Tipos de amamentações } \\
\cline { 2 - 3 } Meses de Lactação & AFI & AFC & ANF \\
\cline { 2 - 3 } & & Freqüência (Episódios/dia/pasto) \\
\hline 1 & $1,06 \pm 0,48^{*}$ & $0,66 \pm 0,32$ & $0,63 \pm 0,28$ \\
2 & $0,97 \pm 0,36^{*}$ & $0,67 \pm 0,15$ & $0,67 \pm 0,15$ \\
3 & $0,86 \pm 0,36^{*}$ & $0,71 \pm 0,31$ & $0,67 \pm 0,22$ \\
4 & $0,80 \pm 0,32^{*}$ & $0,67 \pm 0,22$ & $0,66 \pm 0,21$ \\
5 & $0,87 \pm 0,40^{*}$ & $0,68 \pm 0,22$ & $0,66 \pm 0,21$ \\
7 & $0,84 \pm 0,41^{*}$ & $0,69 \pm 0,24$ & $0,65 \pm 0,21$ \\
8 & $0,77 \pm 0,27^{*}$ & $0,70 \pm 0,21$ & $0,65 \pm 0,21$ \\
9 & $0,81 \pm 0,40$ & $0,68 \pm 0,27$ & $0,65 \pm 0,23$ \\
\hline
\end{tabular}

* indica nível de significância entre AFI e os demais tipos de amamentações $(\mathrm{P}<0,05)$. 
Tabela 2. Médias e respectivos desvios-padrão da duração (segundos/dia/pasto) das amamentações cedidas pelas fêmeas para os diferentes tipos de amamentação, em relação aos meses de lactação. (AFI - amamentação filial isolada; AFC - amamentação filial coletiva e ANF - amamentação não-filial)

\begin{tabular}{|c|c|c|c|}
\hline \multirow{3}{*}{ Meses de Lactação } & \multicolumn{3}{|c|}{ Tipos de amamentações } \\
\hline & AFI & AFC & ANF \\
\hline & \multicolumn{3}{|c|}{ (Segundos/dia/pasto) } \\
\hline 1 & $20,48 \pm 11,9 *$ & $3,90 \pm 8,74$ & $1,17 \pm 1,55$ \\
\hline 2 & $13,19 \pm 9,81^{*}$ & $1,54 \pm 3,98$ & $0,67 \pm 0,15$ \\
\hline 3 & $9,44 \pm 10,17^{*}$ & $2,96 \pm 7,09$ & $1,31 \pm 2,66$ \\
\hline 4 & $6,83 \pm 7,86^{*}$ & $1,58 \pm 5,08$ & $0,90 \pm 1,52$ \\
\hline 5 & $10,20 \pm 11,71^{*}$ & $2,80 \pm 4,99$ & $0,99 \pm 1,84$ \\
\hline 6 & $8,11 \pm 10,9^{*}$ & $2,84 \pm 5,34$ & $0,71 \pm 0,71$ \\
\hline 7 & $7,76 \pm 7,76^{* *}$ & $1,85 \pm 3,49$ & $0,83 \pm 1,12$ \\
\hline 8 & $5,75 \pm 9,69 * *$ & $2,26 \pm 3,95$ & $1,02 \pm 1,26$ \\
\hline 9 & $0,61 \pm 0,26$ & $0,61 \pm 0,26$ & $0,61 \pm 0,26$ \\
\hline
\end{tabular}

Tabela 3. Médias e respectivos desvios-padrão da freqüência (episódios/dia/pasto) das mamadas executas pelos bezerros para os diferentes tipos, em relação à idade dos bezerros. (MFI - mamada filial isolada; MFC1 - mamada filial coletiva na própria mãe; MFC2 - mamada filial coletiva em outras fêmeas e MNF - mamada não-filial)

\begin{tabular}{|c|c|c|c|c|}
\hline \multirow{3}{*}{$\begin{array}{l}\text { Idade dos Bezerros } \\
\text { (Meses) }\end{array}$} & \multicolumn{4}{|c|}{ Tipos de mamadas } \\
\hline & \multicolumn{4}{|c|}{ Freqüência (Episódios/dia/pasto) } \\
\hline & MFI & MFC1 & MFC2 & MNF \\
\hline 1 & $1,16 \pm 0,48^{*}$ & $0,72 \pm 0,24$ & $0,77 \pm 0,17$ & $0,77 \pm 0,10$ \\
\hline 2 & $1,10 \pm 0,61 *$ & $0,70 \pm 0,27$ & $0,77 \pm 0,20$ & $0,72 \pm 0,06$ \\
\hline 3 & $0,86 \pm 0,32 * *$ & $0,73 \pm 0,29$ & $0,78 \pm 0,20$ & $0,69 \pm 0,11$ \\
\hline 4 & $0,80 \pm 0,32 * * *$ & $0,66 \pm 0,22$ & $0,80 \pm 0,23$ & $0,74 \pm 0,12$ \\
\hline 5 & $0,86 \pm 0,39 * *$ & $0,68 \pm 0,25$ & $0,78 \pm 0,15$ & $0,71 \pm 0,00$ \\
\hline 6 & $0,82 \pm 0,41 * * *$ & $0,69 \pm 0,25$ & $0,80 \pm 0,14$ & $0,73 \pm 0,05$ \\
\hline 7 & $0,72 \pm 0,28$ & $0,67 \pm 0,24$ & $0,74 \pm 0,10$ & $0,71 \pm 0,00$ \\
\hline 8 & $0,81 \pm 0,40$ & $0,67 \pm 0,25$ & $0,72 \pm 0,50$ & $0,71 \pm 0,00$ \\
\hline 9 & $0,60 \pm 0,26$ & $0,60 \pm 0,26$ & $0,71 \pm 0,00$ & $0,71 \pm 0,00$ \\
\hline
\end{tabular}

* indica nível de significância entre MFI e os demais tipos de mamadas $(\mathrm{P}<0,05)$, ** indica nível de significância entre MFI e MFC1 e MNF $(\mathrm{P}<0,05)$ e $* * *$ indica nível de significância entre MFI e MFC1 $(\mathrm{P}<0,05)$.

Tabela 4. Médias e respectivos desvios-padrão da duração (segundos/dia/pasto) das mamadas executas pelos bezerros para os diferentes tipos, em relação à idade dos bezerros. (MFI - mamada filial isolada; MFC1 - mamada filial coletiva na própria mãe; MFC2 - mamada filial coletiva em outras fêmeas e MNF - mamada não-filial)

\begin{tabular}{lcccc}
\hline \multirow{2}{*}{$\begin{array}{c}\text { Idade dos Bezerros } \\
\text { (Meses) }\end{array}$} & \multicolumn{4}{c}{ Tipos de mamadas } \\
\cline { 2 - 4 } & MFI & Muração (Segundos/dia/pasto) & MNF \\
\cline { 2 - 4 } & $20,87 \pm 11,22^{*}$ & $2,97 \pm 6,42$ & $2,63 \pm 5,89$ & $1,56 \pm 1,85$ \\
1 & $15,41 \pm 10,19^{*}$ & $2,83 \pm 4,62$ & $2,96 \pm 6,05$ & $1,23 \pm 2,90$ \\
2 & $9,18 \pm 10,60^{*}$ & $4,75 \pm 9,22$ & $4,48 \pm 6,18$ & $1,04 \pm 2,15$ \\
3 & $7,05 \pm 8,10^{*}$ & $1,58 \pm 5,08$ & $3,52 \pm 7,11$ & $1,46 \pm 3,04$ \\
4 & $10,00 \pm 11,30^{*}$ & $2,50 \pm 4,76$ & $4,15 \pm 7,03$ & $0,71 \pm 0,00$ \\
5 & $7,28 \pm 10,39^{* *}$ & $3,00 \pm 6,00$ & $4,97 \pm 6,24$ & $1,27 \pm 1,83$ \\
6 & $4,38 \pm 7,51^{+}$ & $1,84 \pm 3,45$ & $2,16 \pm 4,44$ & $0,82 \pm 0,65$ \\
7 & $5,88 \pm 9,64$ & $2,72 \pm 4,45$ & $1,42 \pm 2,36$ & $0,71 \pm 0,00$ \\
8 & $0,60 \pm 0,26$ & $0,60 \pm 0,26$ & $0,71 \pm 0,00$ & $0,71 \pm 0,00$ \\
9 & & & MFC2 & \\
\hline
\end{tabular}

* indica nível de significância entre MFI e os demais tipos de mamadas $(\mathrm{P}<0,05)$, ** indica nível de significância entre MFI e MFC1 e MNF $(\mathrm{P}<0,05)$ e ${ }^{+}$indica nível de significância entre MFI e MFC2 e MNF $(\mathrm{P}<0,05)$. 
mães, os bezerros aumentaram a procura por outras fêmeas, consequentemente adicionando os valores da MFC2. Dessa forma, os bezerros são motivados a procurar outras fêmeas, principalmente quando percebem que algumas estão amamentando, então ele é estimulado, aumentando, portanto, as tentativas para mamar nessas fêmeas, cabendo a elas permitirem ou não a aloamamentação.

Em relação ao sexo, não houve diferenças significativas $(\mathrm{P}>0,05)$ para as médias da frequência e a duração dos tipos de mamadas executadas pelos bezerros, machos e fêmeas. Os valores da frequência da MFI, MFCI, MFC2 e MNF executada pelos machos foram 0,91 \pm 0,29; 0,79 \pm 0,19; 0,80 \pm 0,02; e 0,71 $\pm 0,05$ episódio/dia/pasto, respectivamente, e para as fêmeas foram $0,87 \pm 0,24 ; 0,72 \pm 0,05$; $0,75 \pm 0,12$; e $0,72 \pm 0,06$ episódio/dia/pasto, respectivamente. A duração das mamadas executadas pelos machos da MFI (8,29 \pm 10,22 segundos/dia/pasto), MFC1 (3,9 \pm 7,43 segundos/dia/pasto), MFC2 (4,41 \pm 7,28 segundos/ dia/pasto) e MNF (0,94 $\pm 1,18$ segundos/dia/pasto) não apresentou diferença quando comparadas com as mamadas executadas pelas fêmeas (MFI: 8,23 \pm 0,14; MFC1: 1,62 $\pm 3,54$; MFC2: 2,59 \pm 4,34; e MNF: $1,04 \pm 1,97$ segundos/dia/pasto, respectivamente)

Contrastando com Andriolo (1995) e Paranhos da Costa et al. (2000), que observaram em bezerros machos quando comparados com as bezerras fêmeas aumento na duração das MFI e MFC2, e que essas, por sua vez, gastam mais tempo com a MFC1, além disso, o ganho de peso dos bezerros machos foi influenciado pela MFI, enquanto para as fêmeas a MCF demonstrou ter maior importância.

A possibilidade de maior investimento materno em filhotes machos em relação às fêmeas foi discutida por Redondo et al. (1992), esses resultados não permitem discutir essa questão, uma vez que não se encontrou diferença em relação aos tipos de mamadas entre bezerros, machos e fêmeas. Segundo Andriolo (1995), as diferenças intersexuais encontradas na evolução temporal de alguns comportamentos ou as diferenças individuais podem estar, provavelmente, ligadas à estruturação social e a diferenças fisiológicas no desenvolvimento do bezerro.

\section{CONCLUSÕES}

As fêmeas bubalinas têm maior instinto materno para a amamentação filial isolada, sendo predominante a frequência e duração desse tipo de amamentação durante quase todo o período de lactação. Os bezerros são mais dependentes da mamada filial isolada nos primeiros cinco meses de idade, após esse período tornam-se mais independentes, adicionando os outros tipos de mamada. O sexo dos bezerros não influenciou nos tipos de mamadas.

\section{AGRADECIMENTOS}

Ao Sr. Aloísio Siqueira de Almeida, proprietário da Fazenda Cataia, e a seus auxiliares, por ter cedido a fazenda para a realização do experimento, e ao suporte financeiro da FAPERJ $n^{\circ}$ E-26/170.645/2003.

\section{REFERÊNCIAS}

Andriolo A (1995) Amamentação coletiva em búfalos (Bubalus bubalis): ontogenia e diferenças individuais. Dissertação de Mestrado. Faculdade de Medicina de Ribeirão Preto-USP, Ribeirão Preto. 145p.

Andriolo A, Paranhos da Costa MJR \& Schmidek, WR (2001) Suckling behaviour in water buffalo (Bubalus bubalis): development and individual differences. Revista de Etologia, 3:129-136.

Bastos R (2000) Amamentação em bubalinos (Bubalus bubalis): um estudo comportamental e endocrinológico. Tese de Doutorado. Faculdade de Medicina de Ribeirão Preto-USP, Ribeirão Preto. 135p.

Bastos R, Paranhos da Costa MJR \& Antunes-Rodrigues J (2002) Comparison of the sucking behaviour of buffaloes on pasture and under confinement. In: First buffalo symposium of Americas, Belém. Anais. p.499-501.

Blass EM \& Teicher MH (1980) Suckling. Science, 210:15-22.

Brown RE (1998) Hormônios e comportamento parental In: Paranhos da Costa MJR e Cromberg VU (Eds). Comportamento Materno em mamíferos (Bases Teóricas e Aplicações aos Ruminantes Domésticos). Ribeirão Preto, Legis Summa. p.54-99.

Hoogland JL, Tamarim RH \& Levy CK (1989) Communal nursing in prairie dogs. Behavioral Ecology and Sociobiology, 24:91-95.

Landete-Castillejos T, Garcia A, Garde J \& Gallego L (2000) Milk intake production curves and allosuckling in captive Iberian red deer, Cervus elaphus hispanicus. Animal Behaviour, 60:679-687.

Martin P \& Bateson P (1986) Measuring Behaviour and introductory guide. Cambridge, Cambridge University Press. 200p.

Murphey RM, Paranhos da Costa MJR, Gomes da Silva R \& Souza RC (1995) Allonursing in river buffalo, Bubalus bubalis: nepotism, incompetence, or thievery? Animal Behaviour, 49:1611-1616.

Murphey RM, Paranhos da Costa MJR, Lima LOS \& Duarte FAM (1991) Communal sucking in water buffalo (Bubalus bubalis). Applied Animal Behaviour Science, 28:341-352.

Nogueira SSC, Otta E, Dias CTS \& Nogueira-Filho SLG (2000) Alloparental behavior in the capybara (Hydrochoerus hydrochaeris). Revista de Etologia, 2:17-21.

Paranhos da Costa MJR \& Andriolo A (1998) Amamentação e Alo-amamentação em Búfalos (Bubalus bubalis). In: Paranhos da Costa MJR e Cromberg VU. (Eds). Comportamento Materno em mamíferos (Bases Teóricas e Aplicações aos Ruminantes Domésticos). Ribeirão Preto, Legis Summa. p.247-261.

Paranhos da Costa MJR, Andriolo A, Oliveira JFS \& Schmidek WR (2000) Suckling and allosuckling in river buffalo calves and its relation with weight gain. Applied Animal Behaviour Science, 66:1-10

Redondo T, Gomendio M \& Medina R (1992). Sex-biased parentoffspring conflict. Behaviour, 123:3-4.

Riedman ML (1982) The evolution of alloparental care and adoption in mammals and birds. The Quartely Review of Biology, 57:405-435. 
Riedman ML \& Le Boeuf B J (1982). Mother-pup separation and adoption in northern elephant seals. Behavioral Ecology and Sociology, 11:203-215.

SAS (1996) Statistical Analysis System Institue. Inc, Cary, NY.

Smith JM (1977) Parental investiment: A prospective analysis. Animal Behaviour, 25:1-9.

Tulloch DG (1979) The water buffalo, Bubalus bubalis, in Australia: Reproductive and parent-offspring behaviour. Australian Wildlife Research, 6:265-287.

Víchová L \& Bartoš L (2005) Allossuckling in cattle: Gain or compensation? Applied Animal Behaviour Science, 94:223-235. 